\title{
Anti-Osteoporotic Effects of Kukoamine B in Osteoblast and Osteoclast Cells and Ovariectomized Mice
}

Eunkuk Park ${ }^{1,2,3 \dagger}$, Jeonghyun Kim ${ }^{1,2 \dagger}$, Mun-Chang Kim ${ }^{1}$, Subin Yeo $^{1,2,3}$, Jieun Kim ${ }^{1,2}$, Chun Whan Choi ${ }^{4}$, Hyun-Seok Jin ${ }^{5}$, Sang Woo Lee ${ }^{6}$, Wan Yi Li ${ }^{7}$, Ji-Won Lee ${ }^{8}$, Jin-Hyok Park ${ }^{9}$, Dam Huh $^{9, *}$ and Seon-Yong Jeong ${ }^{1,2 *}$

${ }^{1}$ Department of Medical Genetics, Ajou University School of Medicine, Suwon 16499, Republic of Korea

${ }^{2}$ Department of Biomedical Sciences, Ajou University Graduate School of Medicine, Suwon 16499, Republic of Korea

${ }^{3}$ Nine B Company, Daejeon 34121, Republic of Korea

${ }^{4}$ Natural Products Research Institute, Gyeonggi Institute of Science \& Technology

Promotion, Suwon 16229, Republic of Korea

${ }^{5}$ Department of Biomedical Laboratory Science, College of Life and Health Sciences, Hoseo University, Asan 31499, Korea

${ }^{6}$ International Biological Material Research Center, Korea Research Institute of Bioscience and Biotechnology, Daejeon 34141, Republic of Korea

${ }^{7}$ Institute of Medicinal Plants, Yunnan Academy of Agricultural Sciences, Kunming 650200, China

${ }^{8}$ Korea Food Research Institute, Seongnam 13539, Republic of Korea

${ }^{9}$ Dongwoodang Pharmacy Co., Ltd., Yeongchen 38819, Republic of Korea

†These authors contributed equally to this work.

*Correspondence: jeongsy@ajou.ac.kr (S-Y.J.); herbleader@omniherb.com (D.H.); +82-31219-4520 (S-Y.J.); Tel.: +82-54-335-8083 (D.H.); +82-31-219-4521(S-Y.J.); Fax: +82-53818-1913 (D.H.) 


\begin{abstract}
:
Osteoporosis is an abnormal bone remodeling condition characterized by decreased bone density, which leads to high risks of broken bones. Previous studies have demonstrated that Lycii Radicis Cortex (LRC) extract inhibits bone loss in ovariectomized (OVX) mice by enhancing the osteoblast differentiation. A bioactive compound, Kukoamine B (KB), was identified from a fractionation of LRC extract as a candidate component responsible for an anti-osteoporotic effect. This study investigated the anti-osteoporotic effects of KB using in vitro and in vivo osteoporosis models. $\mathrm{KB}$ treatment significantly increased the osteoblastic differentiation and mineralized nodule formation of osteoblastic MC3T3-E1 cells, while it significantly decreased the osteoclast differentiation of primary-cultured monocytes derived from mouse bone marrow. The effects of KB on osteoblastic and osteoclastic differentiations under more physiological conditions were also examined. In the co-culture of MC3T3-E1 cells and monocytes, $\mathrm{KB}$ promoted osteoblast differentiation but did not affect osteoclast differentiation. For the in vivo experiments, $\mathrm{KB}$ significantly inhibited OVX-induced bone mineral density loss and restored the impaired bone structural properties in osteoporosis model mice. These results suggest that KB may be a potential therapeutic candidate for the treatment of osteoporosis.
\end{abstract}

Keywords: osteoporosis; herbal medicine; Kukoamine B; osteoblast; osteoclast; bone mineral density; ovariectomized mice 


\section{Introduction}

Bone is a living organ that is constantly remodeled and is maintained by a balance between bone formation and resorption [1,2]. Bone remodeling is a physiological condition that is regulated by replacing new bone formations by osteoblasts and removing old or damaged bone by osteoclasts [1]. Osteoblasts, which are differentiated from mesenchymal stem cells, play an important role in bone formation by synthesizing various bone matrix proteins and transporting mineral into the matrix. Osteoclasts are differentiated from mononuclear cells of the monocyte/macrophage lineage, and they break down bone tissue that is essential for osteoblast new bone formation. Bone formation includes the proliferation and differentiation of osteoblasts via the activation of alkaline phosphatase (ALP), collagen synthesis, and the mineralization of bone [3]. Bone resorption involves the differentiation of osteoclasts via the activation of tartrate-resistant acid phosphatase (TRAP) [4, 5]. The balanced relationship between bone formation and bone resorption is critical for maintaining bone strength and preventing bone loss [6]; however, an imbalanced regulation of these bone-remodeling processes leads to serious bone loss with metabolic bone disease, called osteoporosis [1]. Osteoporosis is a serious condition that causes a loss of bone density and strength, leading to increased painful bone fractures [7]. Osteoporotic fractures become a serious health problem, particularly for aging postmenopausal women [8]. Currently, various pharmacological treatments for osteoporosis therapies are proven to reduce fracture risks, such as inhibitors of bone resorption by osteoclasts (bisphosphonates), selective estrogen receptor modulators, and the monoclonal antibody to the receptor activator of nuclear factor kappa-B ligand (RANKL) or stimulators of bone formation by cells of the osteoblast lineage (parathyroid hormone analogs) [9].

Natural herbal plants have been widely used for alternative medicinal purposes due to the potential fewer side effects for long-term use [10-12]. Studies have suggested that pharmacological bio-active compounds from plats have beneficial therapeutic effects on herbal medicines $[13,14]$. For the treatment of osteoporosis, many herbal medicines derived from natural products have been reported as having a long tradition of use with fewer negative effects $[10,15]$. In addition, bio-active components and related properties for alternative treatments of osteoporosis have been identified [16-19]. Because long-term treatment is required for 
osteoporosis, herbal medicine is a good therapy for preventing and treating osteoporosis. The natural plant Lycii radicis cortex (LRC) has been used as traditional medicine in East Asia, and physiological bio-active properties, such as apigenin, luteolin, kaempferol, quercetin, oleanolic acid, dihydrophaseic acid, and urosolic acid, were identified in LRC extract [20, 21]. Our previous study suggested that LRC extract promoted osteoblast differentiation and inhibited the loss of bone mineral density (BMD) in an osteoporotic mice model without negative side effects [22]. Furthermore, LRC prevented osteoclast differentiation induced by RANKL through the down-regulation of osteoclastogenesis-related markers [23]. Recently, dihydrophaseic acid 3'-O-beta-d-glucopyranoside was identified as a candidate bioactive compound for the anti-osteoporotic effects of LRC extract [24]; however, because a small amount of this compound is contained in LRC extract, other component(s) responsible for the bone formation enhancing effect of LRC may still be unidentified.

The aim of this study was to identify the other bioactive component(s) for anti-osteoporotic effects in LRC extract. First, a fractionation of the ethanol LRC extract was performed, and then a single compound was isolated. Next, the anti-osteoporotic effects of the compound on in vitro and in vivo osteoporosis models were investigated.

\section{Results and Discussion}

\subsection{A Bioactive Component Promoting Osteoblast Differentiation Was Isolated from LRC Extract}

Our previous study reported that the ethanol extract of LRC induced osteoblast differentiation in preosteoblast MC3T3-E1 cells and prevented the loss of bone mineral density in ovariectomized (OVX) mice [22]. The study identified the 13 main constituents using a highperformance liquid chromatography (HPLC)-electrospray ionization (ESI)-tandem mass spectrometry system [22]. In the present study, a fractionation of the bioactive component from LRC ethanol was performed (Figure S1). Seventy percent of LRC ethanol extract was fractionated, and each fraction was investigated for a bone formation-enhancing effect using an alkaline phosphatase (ALP) activity assay in preosteoblast MC3T3-E1 cells for identifying the bio-active fraction. ALP is a homodimeric protein enzyme located in the cell membrane of 
osteoblasts and is a reliable marker of bone metabolism during osteoblast differentiation [25]. Therefore, an ALP assay was conducted to screen the bioactive fraction on bone formation. Each fraction was treated in an osteoblastic cell line for three days, and osteoblast differentiation was measured by the ALP assay. Consequently, a bioactive single compound, Kukoamine B (KB), was isolated from LRC extract and identified by nuclear magnetic resonance (NMR) and mass spectrometry analyses (Figure 1 and Figure S2).

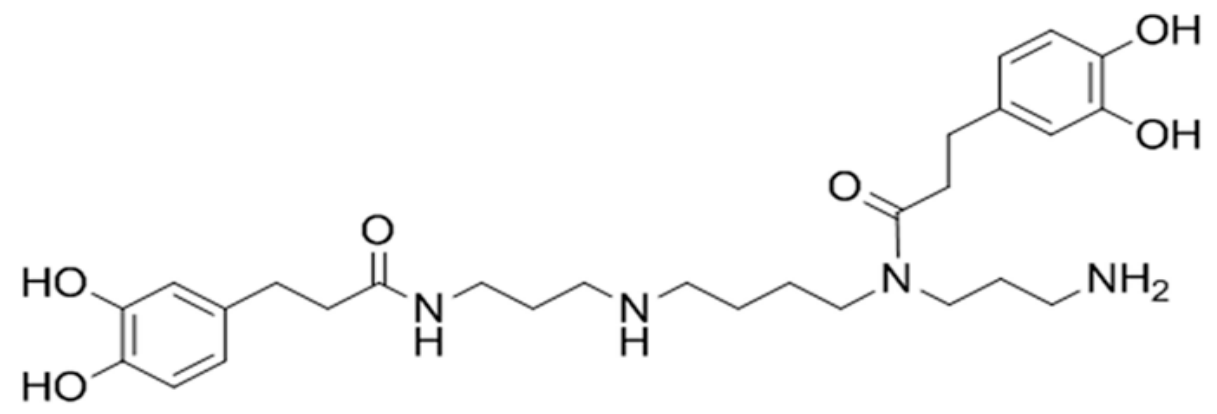

Figure 1. Chemical structure of the isolated Kukoamin B.

$\mathrm{KB}$ is a natural spermine alkaloid compound that was first isolated from LRC [26]. It has been reported that KB neutralizes both lipopolysaccharides (LPS) and oligodeoxynucleotides containing $\mathrm{CpG}$ motifs (CpG DNA) and inhibits LPS- or CpG DNA-mediated proinflammatory signal transduction and cytokine expression [27, 28]. The biological characteristics of $\mathrm{KB}$ on osteoporosis have not been studied.

\subsection{KB Increased Osteoblast Differentiation and the Mineralized Nodule Formation of} Preosteoblastic MC3T3-E1 Cells

First, the effects of $\mathrm{KB}$ on osteoblast differentiation in the MC3T3-E1 cell line were examined. Osteoblast differentiation is characterized by three stages: (1) cell proliferation, (2) matrix maturation, and (3) matrix mineralization [29]. To evaluate the effect of $\mathrm{KB}$ on osteoblast differentiation, orthodox methods were used, including cellular proliferation, ALP activity, and mineralization. ALP activity plays a crucial role in the mineralization of newly formed bone and increases during osteoblast differentiation [30, 31]. Cells were cultured with three different concentrations of $\mathrm{KB}(5,10$, and $20 \mu \mathrm{M})$, and ALP activity was assessed at 2, 3, 
4, and 5 days (Figure S3). ALP activities in the KB treated cells ( 5 and $10 \mu \mathrm{M})$ were increased at 3 and 4 days, but they decreased at day 5 (Figure S3). After 5 days incubation with KB (5, 10 , and $20 \mu \mathrm{M}$ ), cell viability was analyzed using a water-soluble tetrazolium salt (WST) assay. A higher ALP activity was observed for the treatment of 10 and $20 \mu \mathrm{M} \mathrm{KB}$ with increased ALP positive staining colonies in osteoblastic MC3T3-E1 cells compared to the control group (Figures $2 \mathrm{~A}$ and $2 \mathrm{C}$ ). $\mathrm{KB}$ of 5,10 , and $20 \mu \mathrm{M}$ did not affect the proliferation of osteoclastic cells (Figure 2B).

A

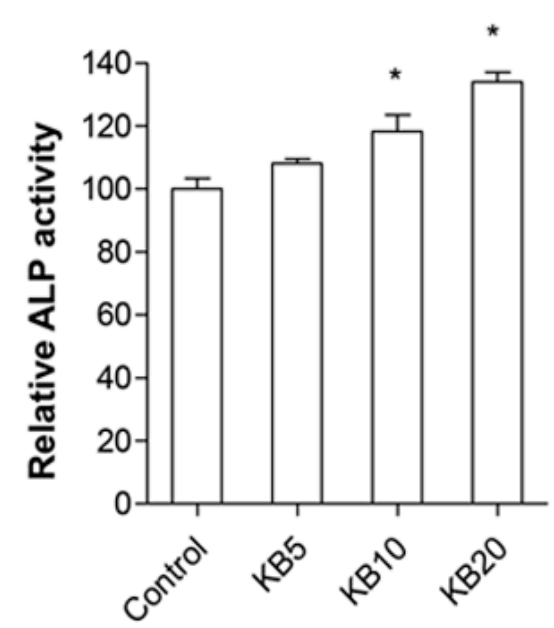

B

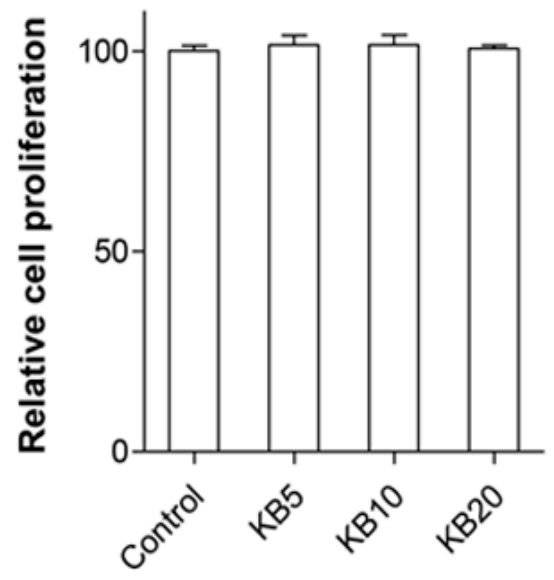

C
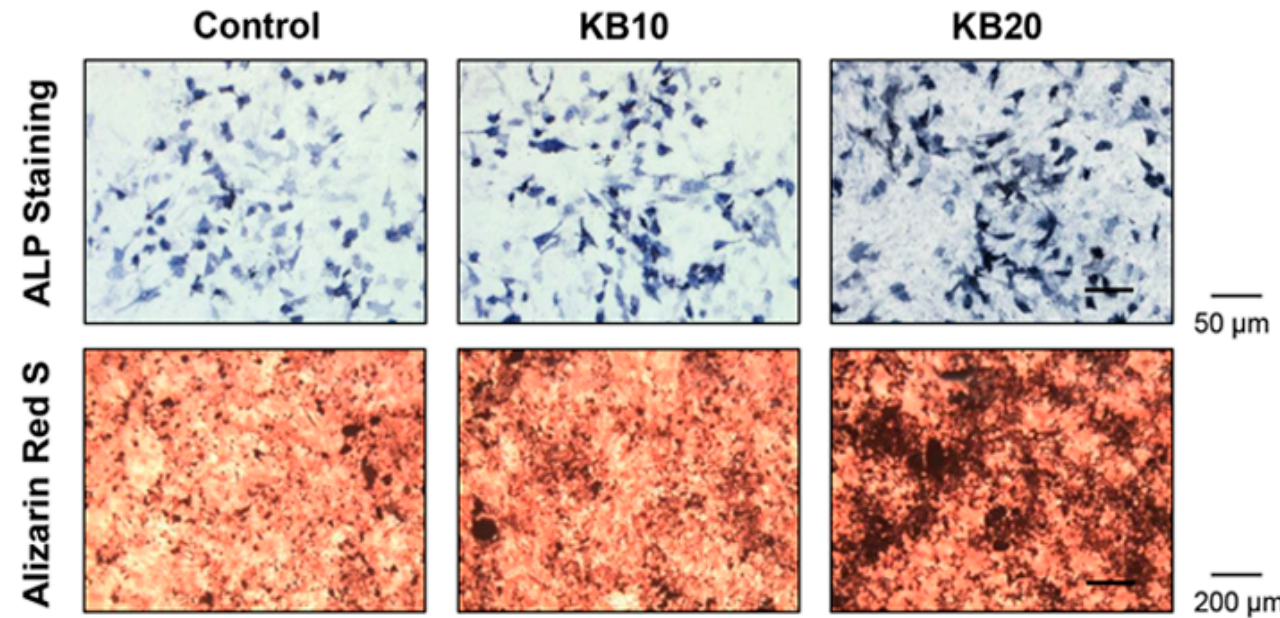

$\overline{200 \mu \mathrm{m}}$

Figure 2. Effects of Kukoamin $B(\mathrm{~KB})$ on cellular differentiation, proliferation, and mineralized nodule formation of the preosteoblast MC3T3-E1 cells. (A) Assessment of the alkaline phosphatase (ALP) activity in the KB-treated MC3T3-E1 cells. After induction of osteoblast differentiation with $50 \mu \mathrm{g} / \mathrm{mL}$ of ascorbic acid and $10 \mathrm{mM}$ of $\beta$ - 
glycerophosphate, cells were treated with three different concentrations of $\mathrm{KB}(5,10$, and $20 \mu \mathrm{M}$ ) for three days, and then ALP activity was assessed. *: $p<0.05$ vs. Control. (B) Assessment of the cellular proliferation in the KB-treated MC3T3-E1 cells. Cells were treated with three different concentrations of $\operatorname{KB}(5,10$, and $20 \mu \mathrm{M})$ for three days, and then cell viability was assessed. (C) Assessment of ALP staining and in vitro bone mineralization in the KB-treated MC3T3-E1 cells. After induction of osteoblast differentiation, cells were treated with 10 and $20 \mu \mathrm{M}$ of KB for three days (for ALP staining) or 21 days (for mineralized nodule formation staining), and then cells were stained with ALP and alizarin red S. The positively stained cells and nodules were visualized under a microscope. Control: KB non-treated cells.

Next, the effects of KB on mineralized nodule formation in MC3T3-E1 cells were examined. Bone matrix is mineralized by osteoblast differentiation, leading to the induction of calcium and phosphate-based minerals. Consequently, bone mineralization with several matrix proteins develops [32]. Many studies have suggested that the mineralized osteoblast is a characteristic method for testing the effects of drug treatments on calcium deposition and bone formation [33, 34]. Alizarin red S is a histochemical method that is commonly used for evaluating calcium-rich deposits in the mineralization of osteoblast cells [35]. Calcium phosphate and osteoblast mineralization with positive alizarin red $\mathrm{S}$ staining revealed successful mineralized osteoblast cells in vitro [36]. The MC3T3-E1 cells were treated with osteoblast induction reagents (ascorbic acid and $\beta$-glycerophosphate) for 21 days, resulting in the mineralized nodule formation and the osteoblast mineralization of MC3T3-El cells. Cotreatment of $\mathrm{KB}(10$ and $20 \mu \mathrm{M})$ with induction reagents presented higher positive alizarin red $\mathrm{S}$ staining colonies than the non-treated control cells. These results demonstrated that KB enhanced the differentiation and mineralized nodule formation of bone-forming osteoblasts.

\subsection{KB Decreased Osteoclast Differentiation of Primary-Cultured Monocytes}

The imbalanced regulation of the bone-remodeling process promotes abnormal metabolic 
bone diseases, especially osteoporosis [1]. Osteoporosis, particularly for postmenopausal women, increases the bone resorption process because osteoclast differentiation occurs rather than bone formation, resulting in an enhancement of the risk of bone fragility and fractures [37]. To examine the effects of KB on bone resorption, whether KB promoted the cellular differentiation of osteoclast cells isolated from the bone marrow of six-week-old mice was investigated. Monocytes from mouse bone marrow were successfully isolated and confirmed by a fluorescence-activated cell sorting (FACS) analysis with monocyte-specific surface markers (CD11b antibody) (Figure 3A). Monocytes are capable of osteoclast differentiation under a suitable microenvironment [38]. For the differentiation of primary-cultured monocytes to osteoclasts, cells were treated with macrophage colony-stimulating factor-1 (M-CSF) and the receptor activator of nuclear factor- $\mathrm{\kappa B}$ ligand (RANKL). During a period of osteoclast differentiation, the treatment of $\mathrm{KB}$ did not affect the proliferation of primary-cultured monocytes of mouse bone marrow (Figure S4). After the induction of osteoclast differentiation, $\mathrm{KB}(5,10$, and $20 \mu \mathrm{M})$ was treated in the primary monocytes for 3 and 6 days, and osteoclast differentiation was analyzed by a Tartrate-resistant acid phosphatase (TRAP) activity assay and TRAP staining. The results showed that KB $(10$ and $20 \mu \mathrm{M})$ treatment significantly decreased TRAP activity and TRAP positive staining colonies (Figures 3B and 3C and Figure S5). These results indicated that $\mathrm{KB}$ inhibited the osteoclast differentiation of monocytes from bone marrow. 
A

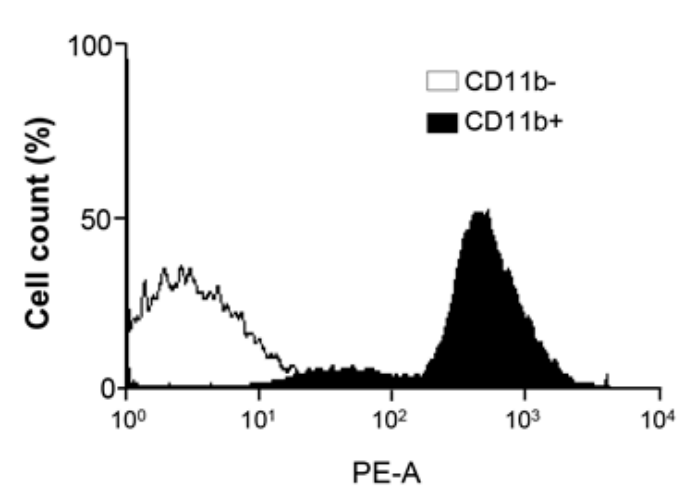

C
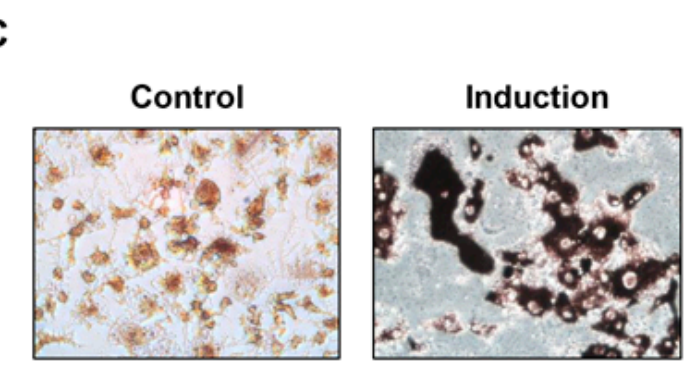

B

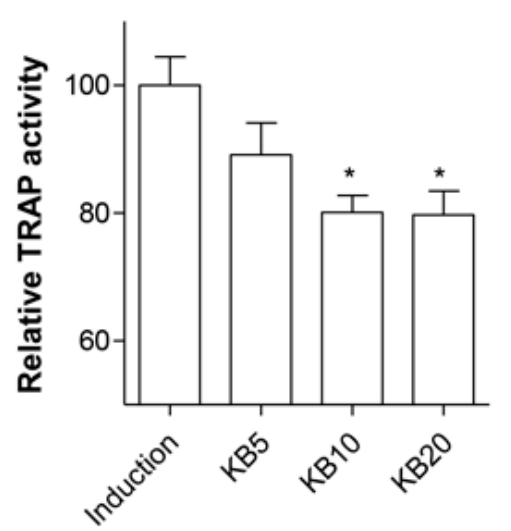

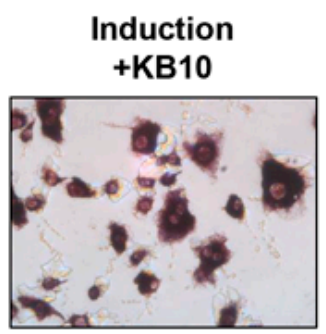

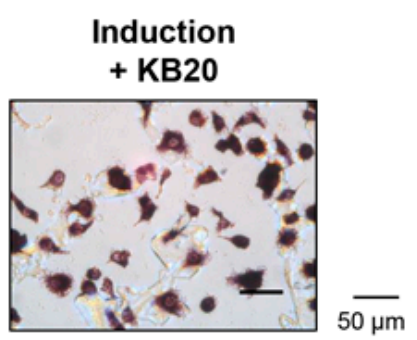

Figure 3. Effects of Kukoamin B (KB) on osteoclast differentiation of primary-cultured monocytes. (A) Validation of successful isolation of monocytes from mouse bone marrow. Primary-cultured monocytes were identified by an immunophenotypic analysis with a monocyte-specific surface positive marker (PE-conjugated CD11b antibody) using a fluorescence-activated cell sorting (FACS) analysis. (B, C) Assessment of tartrateresistant acid phosphatase (TRAP) activity in the KB-treated monocyte cells. After induction of osteoclast differentiation, cells were treated with $\mathrm{KB}(5,10$, and $20 \mu \mathrm{M})$ for 6 days, and then TRAP activity was assessed (B). The cells were also stained with a TRAP staining kit, and the differentiated osteoclast cells were visualized under a microscope (C). Control: non-induction of osteoclast differentiation. Induction: induction of osteoclast differentiation with $30 \mathrm{ng} / \mathrm{mL}$ of a macrophage colony-stimulating factor (M$\mathrm{CSF}$ ) and $50 \mathrm{ng} / \mathrm{ml}$ of a receptor activator of nuclear factor kappa-B ligand (RANKL). All of the experiments were repeated three times. ${ }^{*}: p<0.05$ vs. Induction. 
Bone remodeling is regulated by two processes: bone formation by the differentiation of osteoblasts and bone resorption by the differentiation of osteoclasts in the surface of the bone [32]. The relationship between bone formation and bone resorption includes the interaction of various cell types during bone remodeling [39, 40]. Osteoclast and osteoblast cells communicate with each other through gap junctions [39]. The effects of KB on bone formation under the more physiological conditions of a co-culture system of preosteoblast MC3T3-E1 cells and primary-cultured monocytes were investigated. We established the co-culture system of preosteoblasts and preosteoclasts based on previous co-culture studies [41-43]. The osteoblast differentiation of co-cultured cells was induced by the treatment of ascorbic acid and $\beta$-glycerophosphate for 3 or 6 days, and the effects of co-treated $\operatorname{KB}(5,10$, and $20 \mu \mathrm{M})$ on osteoblast and osteoclast differentiations were examined. KB treatment increased ALP activity compared to the non-treated control cells, and a higher ALP activity was observed at 3 days of $\mathrm{KB}$ treatment rather than 6 days (Figure 4A and Figure S6A); however, KB did not influence TRAP activity in a co-cultured system at either 3 or 6 days of incubation (Figure 4B and Figure S6B).

A

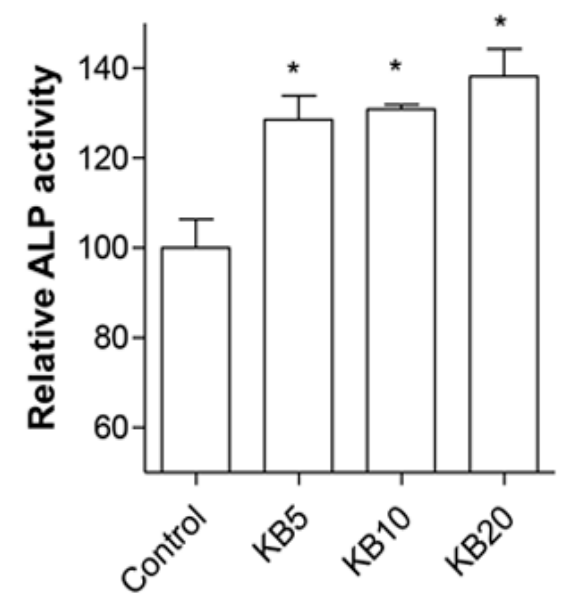

B

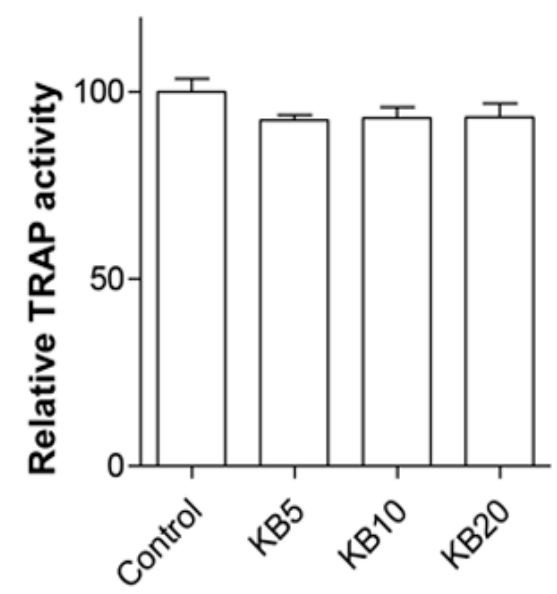

Figure 4. Effects of Kukoamin B (KB) on osteoblast and osteoclast differentiation in the co-culture of preosteoblasts and primary monocytes. Co-cultured MC3T3-E1 and primary monocyte cells were treated with osteoblast differentiation reagents, ascorbic acid and $\beta$ glycerophosphate, and then co-treated with $\mathrm{KB}(5,10$, and $20 \mu \mathrm{M})$ for 3 days. Alkaline 
phosphatase (ALP) activity (A) and tartrate-resistant acid phosphatase (TRAP) activity (B) were assessed in the co-culture cells. Control: KB non-treated cells. *: $p<0.05$ vs. Control.

The homeostasis of bone remodeling is maintained by the balance of bone resorption and bone formation [1]. A previous study demonstrated that a co-culture of preosteoblasts and preosteoclasts enhanced both osteoblast and osteoclast differentiation [41]; however, other studies have suggested that co-cultured cells enhanced osteoblast differentiation but reduced osteoclast differentiation [42, 43]. In the present study, KB treatment promoted osteoblast differentiation but did not affect osteoclast differentiation in the co-culture system, indicating that KB plays a key role in osteoblast differentiation during the bone remodeling process.

\subsection{KB Inhibited BMD Loss in OVX mice}

It is well-known that OVX mice present a reduced bone mass and quality with significantly decreased BMD and bone mineral content (BMC) [44]. Based on the in vitro study, the antiosteoporotic effects of KB in OVX mice was investigated. Seven-week-old female ddy mice underwent either an ovariectomy or sham-operated surgery (Sham). After surgery, the mice were divided into four groups: (1) Sham, (2) OVX control, (3) OVX treated with $2 \mathrm{mg} / \mathrm{kg} / \mathrm{day}$ of KB, and (4) OVX administrated with $5 \mathrm{mg} / \mathrm{kg} /$ day of $\mathrm{KB}$ ( $\mathrm{n}=5$ in each group). The mice were treated for 12 weeks, and the BMD and BMC of the right femur were measured at 0,6 , and 12 weeks using a PIXI-mus bone densitometer (Figure 5A). At the end of the in vivo experiment, transverse microcomputed tomography (micro-CT) images of the right femur were scanned (Fig 5B), and micro-CT images were analyzed, including bone volume (BV/TV), trabecular thickness (Tb.Th), trabecular number (Tb.N), and trabecular spacing (Tb.Sp) (Figure $5 \mathrm{C})$. 
A

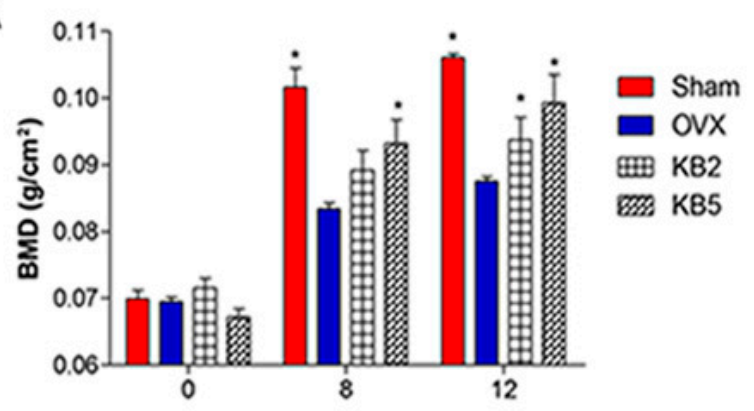

C
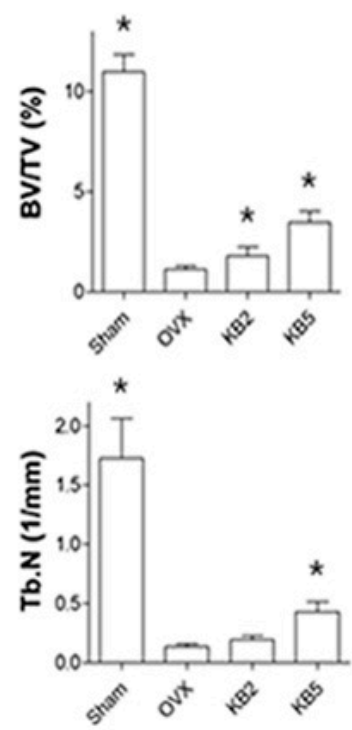
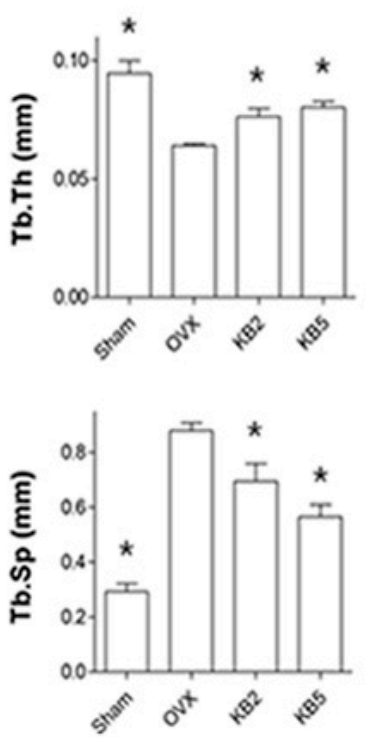

B
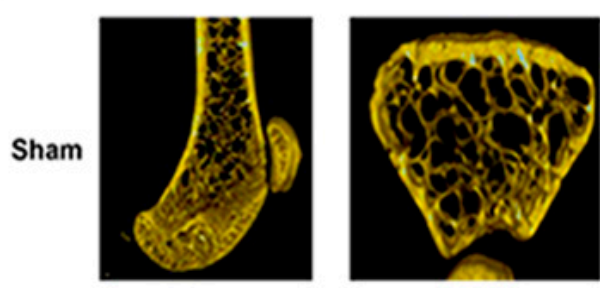

ovx
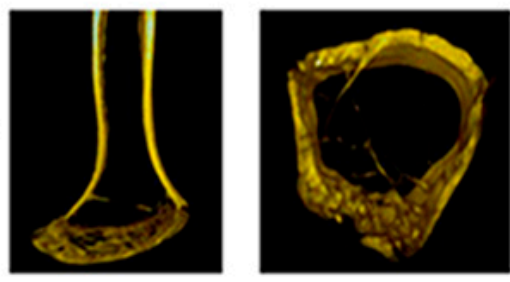

KB2
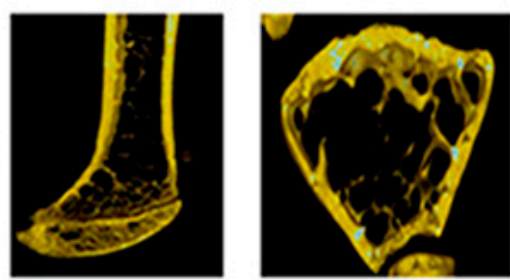

KB5
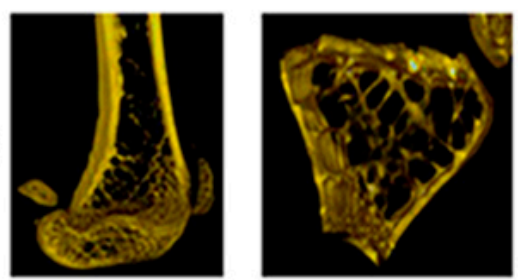

Figure 5. Effects of Kukoamin B (KB) on the improvement of bone mineral density (BMD) and bone structural properties in ovariectomized (OVX) osteoporosis model mice. The OVX mice were administered with KB ( 2 or $5 \mathrm{mg} / \mathrm{kg} /$ day) for 12 weeks. Sham: sham operated group ( $\mathrm{n}=5)$, OVX: KB non-administered mice group (n=5). (A) BMD of right femur was measured using a PIXI-mus bone densitometer at 0,6 , and 12 weeks. (B) Transverse microcomputed tomography (micro-CT) images of the right femur were scanned at the end of the experiment. (C) Trabecular bone structural properties, including bone volume (BV/TV), trabecular thickness (Tb.Th), number (Tb.N), and spacing (Tb.Sp), were analyzed at the end of the experiment. *: $p<0.05$ vs. OVX group.

As expected, OVX mice showed a significantly reduced BMD in the right femur bone and impaired trabecular bone structural properties. The OVX group showed decreased BV/TV, 
Tb.Th, and Tb.N and increased Tb.Sp compared to the Sham group, indicating OVX-induced osteoporotic-bone loss was processed; however, BMC and food intake did not differ among the Sham, OVX control, or KB-administered groups (data not shown). KB administration inhibited the OVX-induced BMD loss in the right femur bone and restored the impaired bone structural properties of BV/TV, Tb.Th, Tb.N, and Tb.Sp compared to the OVX control group (Figure 5). These results demonstrated the anti-osteoporotic effects of $\mathrm{KB}$ in vivo.

\section{Experimental Section}

\subsection{Fractionation, Isolation, and Structure Elucidation of the Bioactive Component}

The dried LRC $(1 \mathrm{~kg})$ was extracted with $70 \%$ ethanol at room temperature, and the ethanol solution was then evaporated under reduced pressure. The $70 \%$ ethanol extract $(97 \mathrm{~g})$ was chromatographed on a Diaion HP-20 gel column and eluted with an $\mathrm{H}_{2} \mathrm{O}$-Ethanol gradient system to obtain four fractions (A-D). Fraction B (11 g) showed that the most potent ALP activity was chromatographed on a RP-18 column $(3 \times 55 \mathrm{~cm})$ and eluted with an $\mathrm{H}_{2} \mathrm{O}$-methanol gradient (from 90:10 to 0:100, $800 \mathrm{~mL}$ for each step) to yield five fractions (B1-B5). Fraction B4 (98 mg) was further chromatographed on a RP-18 column $(2 \times 30 \mathrm{~cm})$ and eluted with $\mathrm{H}_{2} \mathrm{O}-$ methanol (from 90:10 to $0: 100,400 \mathrm{~mL}$ for each step) to obtain a bioactive component (20 $\mathrm{mg}$ ) (Figure S1). The structure of the bioactive component was elucidated by proton nuclear magnetic resonance $\left({ }^{1} \mathrm{H}-\mathrm{NMR}\right)$, carbon-13 nuclear magnetic resonance $\left({ }^{13} \mathrm{C}-\mathrm{NMR}\right)$, and mass spectrometry analyses (Figure S2) as well as by a comparison with the previously reported data [26] (Figure S2).

\subsection{Cell Culture and Reagents}

A mouse MC3T3-E1 cell line was purchased from the RIKEN Cell Bank (Tsukuba, Japan) and cultured in an $\alpha$-modified minimal essential medium ( $\alpha$-MEM) supplemented with 10\% FBS, penicillin $(100 \mathrm{U} / \mathrm{ml})$, and streptomycin $(100 \mu \mathrm{g} / \mathrm{ml})$. Osteoblast differentiation was induced by adding an osteogenic medium containing ascorbic acid $(50 \mathrm{ug} / \mathrm{ml})$ and $\beta$ - 
Glycerophosphate $(10 \mathrm{mM})$ after allowing $24 \mathrm{~h}$ for cell adherence (day 0). For the primaryculture of monocytes, bone marrow cells were flushed from the femoral bones of 6-week-old mice with a fine-bore syringe in the presence of $1 \mathrm{mM}$ ascorbate-2-phosphate (Sigma-Aldrich; St. Louis, USA). The isolated monocyte cells were identified by immunophenotypic analysis with a CD11b antibody (BioLegend; San Diego, USA) using an FACS Aria III cell sorter (BD Biosciences, San Jose, USA) and FACS Diva software (BD Biosciences). Monocyte cells were cultured in an $\alpha$-MEM medium in the presence of $30 \mathrm{ng} / \mathrm{ml}$ of M-CSF (PeproTech; Rocky Hill, USA) and $50 \mathrm{ng} / \mathrm{ml}$ of RANKL (PeproTech) for the induction of differentiation from preosteoclasts to osteoclasts [45]. For the co-culture system, MC3T3-E1 $\left(2 \times 10^{4}\right.$ cells/well) cells and primary monocytes $\left(4 \times 10^{4}\right.$ cells/well) were co-cultured in osteoblast differentiation media containing ascorbic acid $(50 \mu \mathrm{g} / \mathrm{ml})$ and $\beta$-glycerophosphate $(10 \mathrm{mM})$. All cultured cells were incubated in a humidified atmosphere at $37^{\circ} \mathrm{C}$ and $5 \% \mathrm{CO}_{2}$. The cells were used at passages 5-10 after purchase for all experiments. The medium was changed every three days. KB was purchased from Aktin (Cat. No. APC-624) (Chengdu, China).

\subsection{WST Assay and ALP Activity Assay and Staining}

The primary-cultured monocytes and MC3T3-E1 cells $\left(3 \times 10^{3}\right.$ cells/well $)$ were incubated in a 96-well plate overnight and co-treated with different concentrations of $\mathrm{KB}(5,10$, and $20 \mu \mathrm{M})$. Cell viability was determined by a WST assay using an EZ-Cytox Cell Viability Assay Kit (Daeil; Seoul, Korea). A WST solution $(20 \mu 1,5 \mathrm{mg} / \mathrm{ml}$ in phosphate-buffered saline) was added to each well, the cells were incubated for another $4 \mathrm{~h}$, and the media were carefully removed. Formazan crystals were dissolved in acidified isopropyl alcohol $(40 \mathrm{mM} \mathrm{HCl}$ in isopropanol), and their absorbances were measured at $450 \mathrm{~nm}$ and $655 \mathrm{~nm}$ using a microplate reader (BIO-RAD; Hercules, CA, USA). Mouse MC3T3-E1 cells were lysed at $4^{\circ} \mathrm{C}$ in a buffer containing $1 \mathrm{mmol} / 1$ Tris- $\mathrm{HCl}\left(\mathrm{pH} 8.8\right.$ ), $0.5 \%$ Triton $\mathrm{X}-100,10 \mathrm{mmol} / 1 \mathrm{Mg}^{2+}$, and $5 \mathrm{mmol} / \mathrm{L}$ p-nitrophenylphosphate as substrates. After homogenization, ALP activity was measured at the absorbance of $405 \mathrm{~nm}$ (BIO-RAD; Hercules, CA, USA). For ALP staining, cells were fixed in cold 4\% paraformaldehyde for $10 \mathrm{~min}$ and washed with PBS three times. The fixed cells were stained with a BCIP/NBT (Sigma-Aldrich; St. Louis, USA) for $30 \mathrm{~min}$ at room temperature. ALP-positive cells were determined under a light microscope. 


\subsection{Mineralized Nodule Formation in Osteoblast Cells}

Preosteoblast MC3T3-E1 cells were cultured in a 48-well plate overnight and treated with $50 \mu \mathrm{g} / \mathrm{ml}$ of ascorbic acid and $10 \mathrm{mM}$ of $\beta$-glycerophosphate with or without treatment with $\mathrm{KB}(10$ and $20 \mu \mathrm{M})$ for three weeks. The cells were fixed with cold $70 \%$ ethanol for $10 \mathrm{~min}$ at room temperature and washed with water. Calcium deposits in the mineralized cells were determined by Alizarin red S (Sigma-Aldrich; St. Louis, USA) staining. Positive Alizarin red $\mathrm{S}$ staining was determined using a light microscope.

\subsection{Co-Culture System of MC3T3-E1 cells and Primary Monocytes}

For the co-culture system, MC3T3-E1 $\left(2 \times 10^{4}\right.$ cells/well $)$ cells were cultured in a 48-well plate overnight, and the isolated monocytes $\left(4 \times 10^{4}\right.$ cells/well $)$ from the mouse bone marrow were added to the MC3T3-E1 cells and incubated for 1 day. The co-culture of MC3T3-E1 cells and primary-cultured monocyte cells was co-incubated with an osteoblast differentiationinduction media containing ascorbic acid $(50 \mu \mathrm{g} / \mathrm{ml})$ and $\beta$-glycerophosphate $(10 \mathrm{mM})$ with or without treatment with $\mathrm{KB}(10$ and $20 \mu \mathrm{M})$ for either three or six days. The differentiations between osteoblast and osteoclast were measured by ALP activity and TRAP activity, respectively.

\subsection{TRAP Staining and Activity Test}

After the induction of the primary-cultured monocytes, the medium was removed and gently washed with PBS. The differentiated osteoclast cells from the monocytes were measured by a TRAP activity assay and were further stained using an Acid-Phosphatase Kit (SigmaAldrich; St. Louis, USA). The absorbance was measured at $405 \mathrm{~nm}$ (BIO-RAD; Hercules, CA, USA), and TRAP activity was expressed as the percentage of the untreated control. For TRAP staining, the cells were fixed in cold 4\% paraformaldehyde for $10 \mathrm{~min}$ and washed with PBS. The fixed cells were stained with an Acid phosphatase and Leukocyte kit (Sigma-Aldrich; St. Louis, USA), for $2 \mathrm{~h}$ at room temperature. TRAP-positive multinucleated cells containing three or more nuclei were counted under a light microscope.

\subsection{Ovariectomized Osteoporosis Model Mice}


The OVX and sham-operated 8-week-old female ddY mice were purchased from Shizuoka Laboratory Center Inc. (Hamamatsu, Japan). The mice were acclimated for 10 days prior to experimentation. The mice were maintained on a diet (5.0 g/day) of Formula-M07 (Feedlab Co., Ltd., Hanam, Korea) and tap water (15 ml/day). All mice were housed individually in clear plastic cages under controlled temperature $\left(23 \pm 2^{\circ} \mathrm{C}\right)$, humidity $(55 \pm 5 \%)$, and illumination (12hour light/dark cycle). Mice were orally administered KB (2 or $5 \mathrm{mg} / \mathrm{kg} /$ day) for 12 weeks. The BMD and BMC were measured at 0,6 , and 12 weeks during the experiment. The animal research procedures were approved by the Animal Care and Use Committee of the Ajou University School of Medicine (IACUC No. 2014-0066), and all experiments were conducted in accordance with the institutional guidelines established by the committee. All efforts were made to minimize animal suffering and to reduce the number of mice used.

\subsection{Measurement of BMD and Micro-CT Imaging in Bones}

After anesthetization using tiletamine/zolazepam (Zoletil; Virbac Laboratories, Carros, France), the mice were placed on the specimen tray for measurements. The right femur of BMC and BMD were measured using a PIXI-mus bone densitometer with on-board PIXI-mus software (GE Lunar, Madison, USA) for small animals, and it was adjusted in relation to body weight. Transverse micro-CT images of the right femur were scanned using a high-energy spiral scan micro-CT, the BrukermicroCT (Skyscan 1173, Belgium), with a voltage of $60 \mathrm{kV}$, a current of $400 \mathrm{uA}$, an exposure time of $400 \mathrm{~ms}$, a magnification of $\mathrm{M}-\mathrm{H}$, a CCD readout of $1280 \times 1280$, and rotation steps of 360 . Reconstruction was performed using NRecon software (BrukermicroCT). Two-dimensional axial and three-dimensional images were reconstructed for qualitative and quantitative analyses. For the region of interest (ROI) measurements and analyses, axial images were displayed using Inveon Research Workplace (Siemens). Axial reformats were performed to allow slice-by-slice, manual tracing of the contours of the trabecular bone. For the cross-sectional study of the trabecular bone, a region was analyzed approximately $300 \mu \mathrm{m}$ below the growth plate. The analysis provided information about the main histomorphometric parameters, including bone volume (BV/TV, \%), trabecular number (Tb.N, 1/mm), thickness (Tb.Th, mm), and spacing (Tb.Sp, mm).

\subsection{Statistical Analysis}


All the experiments were repeated at least three times unless stated otherwise, and the results were presented as the means \pm standard deviation, as indicated. A statistical software package (SPSS 11.0 for Windows, SPSS Inc., Chicago, IL, USA) was used to perform the statistical tests. The statistical significance of the differences was assessed by the Student's $t$ test. A probability value $(p)$ less than $0.05(p<0.05)$ was considered statistically significant. The results were expressed as mean \pm SEM.

\section{Conclusion}

This is the first study to evaluate the anti-osteoporotic effect of Kukoamine B $(\mathrm{KB})$ isolated from LRC in vitro and in vivo. The in vitro experiments demonstrated that $\mathrm{KB}$ enhanced the differentiation and mineralized nodule formation of preosteoblastic MC3T3-E1 cells and inhibited the osteoclast differentiation of preosteoclast-lineage monocytes isolated from mice bone marrow. In a co-culture of MC3T3-E1 cells and primary-cultured monocytes, $\mathrm{KB}$ treatment promoted osteoblast differentiation but not osteoclast differentiation. The in vivo experiments demonstrated that KB significantly improved OVX-induced bone mineral density loss and impaired bone structural properties in osteoporosis model mice. The results suggest that KB may be a good therapeutic candidate for the treatment and prevention of osteoporosis.

\section{Acknowledgements}

This study was supported by the High Value-Added Food Technology Development Program, Ministry of Agriculture, Food and Rural Affairs, Republic of Korea (115007-03-2HD020 and 117041-03-1-SB010) and the National Research Foundation of Korea (NRF) grant funded by the Korean government (2016K1A1A8A01939208).

\section{Author Contribution}

S-Y.J., E.P., J.K., and H.D. designed the research. E.P., J.K., M-C.K., S.Y., J.K., C.W.C., H-S. J., J-W.L, and J-H.P. performed the research and analyzed the data. S.W.L., W.Y.L., H.D., and S-Y.J. interpreted the data. E.P., J.K., and S-Y.J. wrote the paper, and S-Y.J. and E.P. edited 
the manuscript. All authors read and approved the final manuscript.

\section{Conflict of Interest}

The authors declare no conflict of interest. 


\section{References}

1. Feng, X.; McDonald, J. M., Disorders of bone remodeling. Annu Rev Pathol 2011, 6, 121-45.

2. Raggatt, L. J.; Partridge, N. C., Cellular and molecular mechanisms of bone remodeling. J Biol Chem 2010, 285, 25103-8.

3. Fakhry, M.; Hamade, E.; Badran, B.; Buchet, R.; Magne, D., Molecular mechanisms of mesenchymal stem cell differentiation towards osteoblasts. World J Stem Cells 2013, 5, (4), 136-48.

4. Boyce, B. F.; Rosenberg, E.; de Papp, A. E.; Duong, L. T., The osteoclast, bone remodelling and treatment of metabolic bone disease. Eur J Clin Invest 2012, 42, (12), $1332-41$.

5. Kikuta, J.; Ishii, M., Osteoclast migration, differentiation and function: novel therapeutic targets for rheumatic diseases. Rheumatology 2013, 52, (2), 226-34.

6. Shieh, A.; Han, W.; Ishii, S.; Greendale, G. A.; Crandall, C. J.; Karlamangla, A. S., Quantifying the Balance Between Total Bone Formation and Total Bone Resorption: An Index of Net Bone Formation. J Clin Endocrinol Metab 2016, 101, (7), 2802-9.

7. Rachner, T. D.; Khosla, S.; Hofbauer, L. C., Osteoporosis: now and the future. Lancet 2011, 377, (9773), 1276-87.

8. Management of osteoporosis in postmenopausal women: 2010 position statement of The North American Menopause Society. Menopause 2010, 17, (1), 25-54; quiz 55-6.

9. Cappola, A. R.; Shoback, D. M., Osteoporosis Therapy in Postmenopausal Women With High Risk of Fracture. JAMA 2016, 316, (7), 715-6.

10. Yuan, H.; Ma, Q.; Ye, L.; Piao, G., The Traditional Medicine and Modern Medicine from Natural Products. Molecules 2016, 21, (5).

11. Barnes, J.; McLachlan, A. J.; Sherwin, C. M.; Enioutina, E. Y., Herbal medicines: challenges in the modern world. Part 1. Australia and New Zealand. Expert Rev Clin Pharmacol 2016, 1-11.

12. Sammons, H. M.; Gubarev, M. I.; Krepkova, L. V.; Bortnikova, V. V.; Corrick, F.; Job, K. M.; Sherwin, C. M.; Enioutina, E. Y., Herbal medicines: challenges in the modern world. Part 2. European Union and Russia. Expert Rev Clin Pharmacol 2016, 1-11.

13. Zhao, S.; Baik, O. D.; Choi, Y. J.; Kim, S. M., Pretreatments for the efficient extraction 
of bioactive compounds from plant-based biomaterials. Crit Rev Food Sci Nutr 2014, 54, (10), 1283-97.

14. Sasidharan, S.; Chen, Y.; Saravanan, D.; Sundram, K. M.; Yoga Latha, L., Extraction, isolation and characterization of bioactive compounds from plants' extracts. Afr J Tradit Complement Altern Med 2011, 8, (1), 1-10.

15. Rodrigues, T.; Reker, D.; Schneider, P.; Schneider, G., Counting on natural products for drug design. Nat Chem 2016, 8, (6), 531-41.

16. Mukwaya, E.; Xu, F.; Wong, M. S.; Zhang, Y., Chinese herbal medicine for bone health. Pharm Biol 2014, 52, (9), 1223-8.

17. Zhang, N. D.; Han, T.; Huang, B. K.; Rahman, K.; Jiang, Y. P.; Xu, H. T.; Qin, L. P.; Xin, H. L.; Zhang, Q. Y., Traditional Chinese Medicine formulas for the treatment of osteoporosis: Implication for antiosteoporotic drug discovery. J Ethnopharmacol 2016, 2, (189), 61-80

18. Che, C. T.; Wong, M. S.; Lam, C. W., Natural Products from Chinese Medicines with Potential Benefits to Bone Health. Molecules 2016, 21, (3), 239.

19. Li, T. M.; Huang, H. C.; Su, C. M.; Ho, T. Y.; Wu, C. M.; Chen, W. C.; Fong, Y. C.; Tang, C. H., Cistanche deserticola extract increases bone formation in osteoblasts. The J Pharm Pharmacol 2012, 64, (6), 897-907.

20. Potterat, O., Goji (Lycium barbarum and L. chinense): Phytochemistry, pharmacology and safety in the perspective of traditional uses and recent popularity. Planta medica 2010, 76, (1), 7-19.

21. Jeong, J. C.; Kim, S. J.; Kim, Y. K.; Kwon, C. H.; Kim, K. H., Lycii cortex radicis extract inhibits glioma tumor growth in vitro and in vivo through downregulation of the Akt/ERK pathway. Oncol Rep 2012, 27, (5), 1467-74.

22. Park, E.; Kim, M. C.; Choi, C. W.; Kim, J.; Jin, H. S.; Lee, R.; Lee, J. W.; Park, J. H.; Huh, D.; Jeong, S. Y., Effects of Dihydrophaseic Acid 3'-O-beta-d-Glucopyranoside Isolated from Lycii radicis Cortex on Osteoblast Differentiation. Molecules 2016, 21, (9).

23. Kim, J. H.; Kim, E. Y.; Lee, B.; Min, J. H.; Song, D. U.; Lim, J. M.; Eom, J. W.; Yeom, M.; Jung, H. S.; Sohn, Y., The effects of Lycii Radicis Cortex on RANKL-induced osteoclast differentiation and activation in RAW 264.7 cells. Int J Mol Med 2016, 37 , 
(3), 649-58.

24. Park, E.; Jin, H. S.; Cho, D. Y.; Kim, J.; Kim, M. C.; Chio, C. W.; Lee, J. W.; Park, J. H.; Chung, Y. S.; Huh, D.; Jeong, S. Y., The effect of Lycii Radicis Cortex extract on bone formation in vitro and in vivo Molecules 2014, 19, (12), 19594-19609.

25. Orimo, H.; Shimada, T., The role of tissue-nonspecific alkaline phosphatase in the phosphate-induced activation of alkaline phosphatase and mineralization in SaOS-2 human osteoblast-like cells. Mol Cell Biochem 2008, 315, (1-2), 51-60.

26. Funayama, S.; Zhang, G.-R.; Nozoe, S., Kukoamine B, a spermine alkaloid from Lycium chinense. Phytochemistry 1995, 38, (6), 1529-31.

27. Liu, X.; Zheng, X.; Wang, N.; Cao, H.; Lu, Y.; Long, Y.; Zhao, K.; Zhou, H.; Zheng, J., Kukoamine B, a novel dual inhibitor of LPS and CpG DNA, is a potential candidate for sepsis treatment. Br J Pharmacol 2011, 162, (6), 1274-90.

28. Qin, W. T.; Wang, X.; Shen, W. C.; Sun, B. W., A novel role of kukoamine B: Inhibition of the inflammatory response in the livers of lipopolysaccharide-induced septic mice via its unique property of combining with lipopolysaccharide. Exp Ther Med 2015, 9, (3), 725-732.

29. Rutkovskiy, A.; Stensløkken, K.-O.; Vaage, I. J., Osteoblast Differentiation at a Glance. Med Sci Monit Basic Res 2016, 22, 95-106.

30. Watts, N. B., Clinical utility of biochemical markers of bone remodeling. Clin Chem 1999, 45, (8 Pt 2), 1359-68.

31. Liu, J.; Nam, H. K.; Campbell, C.; Gasque, K. C.; Millan, J. L.; Hatch, N. E., Tissuenonspecific alkaline phosphatase deficiency causes abnormal craniofacial bone development in the Alpl(-/-) mouse model of infantile hypophosphatasia. Bone 2014, 67, 81-94.

32. Raggatt, L. J.; Partridge, N. C., Cellular and molecular mechanisms of bone remodeling. J Biol Chem 2010, 285, (33), 25103-8.

33. Gough, J. E.; Jones, J. R.; Hench, L. L., Nodule formation and mineralisation of human primary osteoblasts cultured on a porous bioactive glass scaffold. Biomaterials 2004, 25, (11), 2039-46.

34. Chou, J.; Hao, J.; Hatoyama, H.; Ben-Nissan, B.; Milthorpe, B.; Otsuka, M., The therapeutic effect on bone mineral formation from biomimetic zinc containing 
tricalcium phosphate (ZnTCP) in zinc-deficient osteoporotic mice. PloS one 2013, 8, (8), e71821.

35. Hoyte, D. A., Alizarin red in the study of the apposition and resorption on bone. Am J Phys Anthropol 1968, 29, (2), 157-77.

36. Virtanen, P.; Isotupa, K., Staining properties of alizarin red S for growing bone in vitro. Acta anatomica 1980, 108, (2), 202-7.

37. Yoshida, K.; Oida, H.; Kobayashi, T.; Maruyama, T.; Tanaka, M.; Katayama, T.; Yamaguchi, K.; Segi, E.; Tsuboyama, T.; Matsushita, M.; Ito, K.; Ito, Y.; Sugimoto, Y.; Ushikubi, F.; Ohuchida, S.; Kondo, K.; Nakamura, T.; Narumiya, S., Stimulation of bone formation and prevention of bone loss by prostaglandin E EP4 receptor activation. Proc Natl Acad Sci USA 2002, 99, (7), 4580-5.

38. Udagawa, N.; Takahashi, N.; Akatsu, T.; Tanaka, H.; Sasaki, T.; Nishihara, T.; Koga, T.; Martin, T. J.; Suda, T., Origin of osteoclasts: mature monocytes and macrophages are capable of differentiating into osteoclasts under a suitable microenvironment prepared by bone marrow-derived stromal cells. Proc Natl Acad Sci USA 1990, 87, (18), 72604.

39. Matsuo, K.; Irie, N., Osteoclast-osteoblast communication. Arch Biochem Biophys 2008, 473, (2), 201-9.

40. Sims, N. A.; Martin, T. J., Coupling the activities of bone formation and resorption: a multitude of signals within the basic multicellular unit. Bonekey Rep 2014, 3, 481.

41. Chen, S.; Ye, X.; Yu, X.; Xu, Q.; Pan, K.; Lu, S.; Yang, P., Co-culture with periodontal ligament stem cells enhanced osteoblastic differentiation of MC3T3-E1 cells and osteoclastic differentiation of RAW264.7 cells. Int J Clin Exp Pathol 2015, 8, (11), 14596-607.

42. Wu, L.; Feyerabend, F.; Schilling, A. F.; Willumeit-Romer, R.; Luthringer, B. J., Effects of extracellular magnesium extract on the proliferation and differentiation of human osteoblasts and osteoclasts in coculture. Acta Biomater 2015, 27, 294-304.

43. Bernhardt, A.; Thieme, S.; Domaschke, H.; Springer, A.; Rosen-Wolff, A.; Gelinsky, M., Crosstalk of osteoblast and osteoclast precursors on mineralized collagen--towards an in vitro model for bone remodeling. J Biomed Mater Res A 2010, 95, (3), 848-56.

44. Ekici, H.; Sontas, B. H.; Toydemir, T. S.; Senmevsim, O.; Kabasakal, L.; Imre, Y., 
Effect of prepubertal ovariohysterectomy on bone mineral density and bone mineral content in puppies. Acta Vet Hung 2005, 53, (4), 469-78.

45. Sun, L.; Peng, Y.; Sharrow, A. C.; Iqbal, J.; Zhang, Z.; Papachristou, D. J.; Zaidi, S.; Zhu, L. L.; Yaroslavskiy, B. B.; Zhou, H.; Zallone, A.; Sairam, M. R.; Kumar, T. R.; Bo, W.; Braun, J.; Cardoso-Landa, L.; Schaffler, M. B.; Moonga, B. S.; Blair, H. C.; Zaidi, M., FSH directly regulates bone mass. Cell 2006, 125, (2), 247-60. 